\title{
Targeting stem cell signaling pathways for drug discovery: advances in the Notch and Wnt pathways
}

\author{
AN Songzhu Michael ${ }^{1 *}$, DING Qiang ${ }^{1}$, ZHANG Jie ${ }^{2}$, XIE Jing Yi $^{2} \&$ LI LingSong ${ }^{2 *}$ \\ ${ }^{1}$ Curegenix Inc., Guangzhou 510633, China; \\ ${ }^{2}$ Center for Stem Cell and NanoMedicine, Shanghai Advanced Research Institute, Chinese Academy of Sciences, \\ Shanghai 201210, China
}

Received February 15, 2014; accepted April 4, 2014; published online May 13, 2014

\begin{abstract}
Signaling pathways transduce extracellular stimuli into cells through molecular cascades to regulate cellular functions. In stem cells, a small number of pathways, notably those of TGF- $\beta$ /BMP, Hedgehog, Notch, and Wnt, are responsible for the regulation of pluripotency and differentiation. During embryonic development, these pathways govern cell fate specifications as well as the formation of tissues and organs. In adulthood, their normal functions are important for tissue homeostasis and regeneration, whereas aberrations result in diseases, such as cancer and degenerative disorders. In complex biological systems, stem cell signaling pathways work in concert as a network and exhibit crosstalk, such as the negative crosstalk between Wnt and Notch. Over the past decade, genetic and genomic studies have identified a number of potential drug targets that are involved in stem cell signaling pathways. Indeed, discovery of new targets and drugs for these pathways has become one of the most active areas in both the research community and pharmaceutical industry. Remarkable progress has been made and several promising drug candidates have entered into clinical trials. This review focuses on recent advances in the discovery of novel drugs which target the Notch and Wnt pathways.
\end{abstract}

stem cell, drug discovery, signaling pathways, Notch, Wnt, cancer

Citation: An SM, Ding Q, Zhang J, Xie JY, Li LS. Targeting stem cell signaling pathways for drug discovery: advances in the Notch and Wnt pathways. Sci China Life Sci, 2014, 57: 575-580, doi: 10.1007/s11427-014-4665-7

Stem cell research has been one of the most exciting fields in biomedicine over the past few years. It has profoundly improved our understanding of fundamental biological processes. The application of stem cells is a promising therapeutic modality, yet, cell therapy has a number of technical challenges and regulatory hurdles to overcome. In contrast, application of the principles of stem cell biology and the use of stem cells themselves as tools in the discovery of novel drugs may generate immediate returns because this approach is mature in terms of technologies and known regulatory pathways. In particular, the targeting of stem cell signaling pathways is proving to be a productive avenue for

*Corresponding author (email: man@ curegenix.com; lils@sari.ac.cn) drug research.

Over the past 30 years, developmental biology studies have revealed a small number of signal transduction pathways involved in stem cell self-renewal and differentiation that govern embryonic development. Among them, signaling cascades elicited by TGF- $\beta / \mathrm{BMP}$, Hedgehog, Notch and Wnt proteins are particularly important. Through intracellular signaling pathways, these secreted proteins control the behavior of recipient stem cells or their differentiated progeny. As a result, the stem cells or differentiated cells undergo phenotypic changes in self-renewal, differentiation, proliferation, or migration. The concerted actions of these signals lead to specific cell fates and the formation of tissues and organs. In adult tissues and organs, the activities of 
these signaling pathways are required to maintain homeostasis of the organism, as well as repair of injuries and regeneration.

From decades of genetic research, the key components of the signaling pathways and the mechanisms by which these signals act in embryonic development have been well-established. By contrast, our understanding of how these signals maintain tissue homeostasis and how their aberrations cause human diseases is still limited. The recent advances in stem cell research, chemical biology, and genomics have now provided the necessary tools to investigate these pathways in a broad range of biological systems and disease models. In particular, there are now unique experimental opportunities to use chemical modulators of these pathways to explore disease mechanisms and assess their therapeutic value.

\section{The Notch pathway}

Among the key signaling pathways that control stem cells in embryonic and adult organisms, the evolutionarily conserved Notch signaling pathway is particularly important for the development and maintenance of the nervous system, muscle, intestine and hematopoiesis. The Notch pathway consists of five known ligands (Jagged-1, -2, Delta-like-1, $-3,-4)$ and four receptors (Notch-1, -2, -3, -4). In the canonical Notch pathway (Figure 1), ligand binding with receptor results in its proteolytic cleavages mediated first by ADAM metalloproteases and then by a $\gamma$-secretase complex. These cleavages of Notch result in the release of a constitutively active Notch intra-cellular domain (NICD) that then translocates to the nucleus where it associates with a larger transcription complex. In net effect, NICD switches the transcriptional complex from a repressed to activated state $[1,2]$.

\section{The Wnt pathway}

The WNT genes were first discovered in Drosophila as wingless [3] and later in mouse tumors as the oncogene int-1 [4] (Figure 2). In humans, the WNT family is composed of 19 lipidated and glycosylated proteins that play essential roles in diverse processes such as stem cell self-renewal, differentiation, cell polarity, proliferation, and migration. Aberrations in the Wnt proteins, or of any of the signaling components in the Wnt pathway, result in abnormal embryonic development or diseases [5].

Wnt signaling encompasses both the canonical $(\beta-$ catenin-dependent) and the non-canonical ( $\beta$-cateninindependent) pathways. The canonical WNT signaling pathway stabilizes $\beta$-catenin and the transcription of genes known to regulate a large array of cellular functions (Figure 2). The non-canonical WNT signaling pathway does not



Figure 1 A diagram of the Notch pathway.

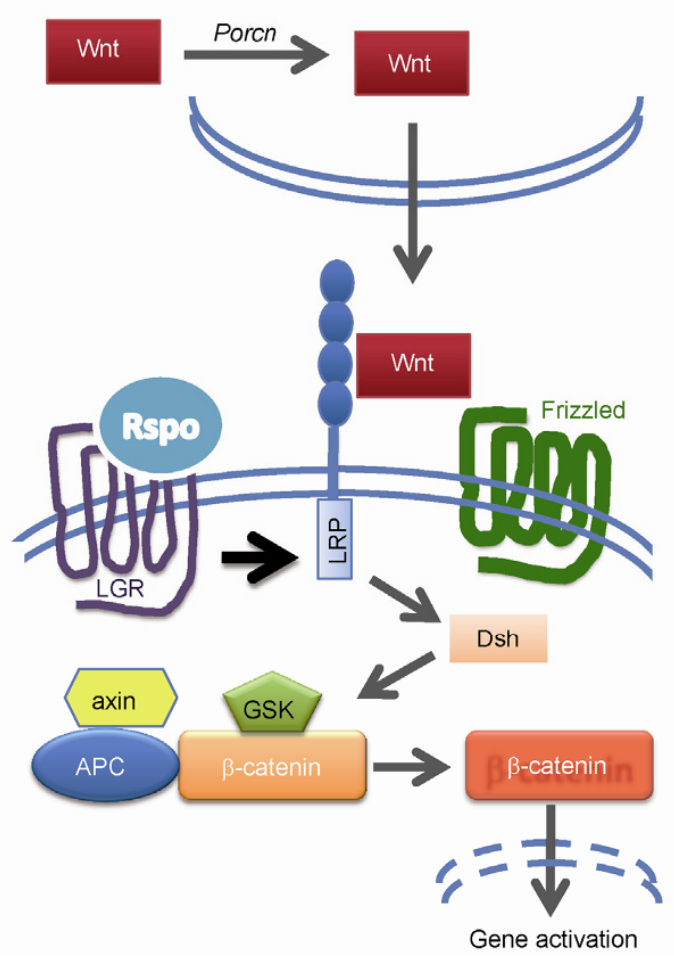

Figure 2 A diagram of the Wnt pathway. 
activate $\beta$-catenin but instead transduces signals to affect cell polarity and migration through the planar cell polarity pathway and GTPases.

\section{Interaction between the Notch and Wnt pathways}

There is an increasing body of evidence that suggests that the stem cell signaling pathways work as a network and exhibit crosstalk (Figure 3). For instance, between WNT and Notch signaling, it was shown in fruit flies that the wingless downstream component disheveled binds to the cytoplasmic tail of Notch [6]. On the other hand, Notch ligand Jagged-1 has been shown to be a Wnt/ $\beta$-catenin target gene in hair follicle formation of the adult epidermis [7]. More recently, Kwon et al. [8] showed a physical interaction between $\beta$-catenin and the cytoplasmic tail of membrane bound Notch in stem and progenitor cells. Thus only the active pool of $\beta$-catenin protein was able to bind Notch. This interaction resulted in degradation of $\beta$-catenin protein, suggesting that a negative interaction exists between the two pathways in these cells. The negative crosstalk between the Notch and Wnt pathways was further demonstrated in keratinocytes. In these cells, inhibition of Notch signaling, through Notch-1 KO, correlated with Wnt activation, whereas overexpression of either NICD or Jagged-1 downregulated the expression of Wnt genes $[9,10]$. These findings have great implication for the therapeutic application of drugs targeting these two pathways, which will be discussed later in light of recent cancer genomic results.

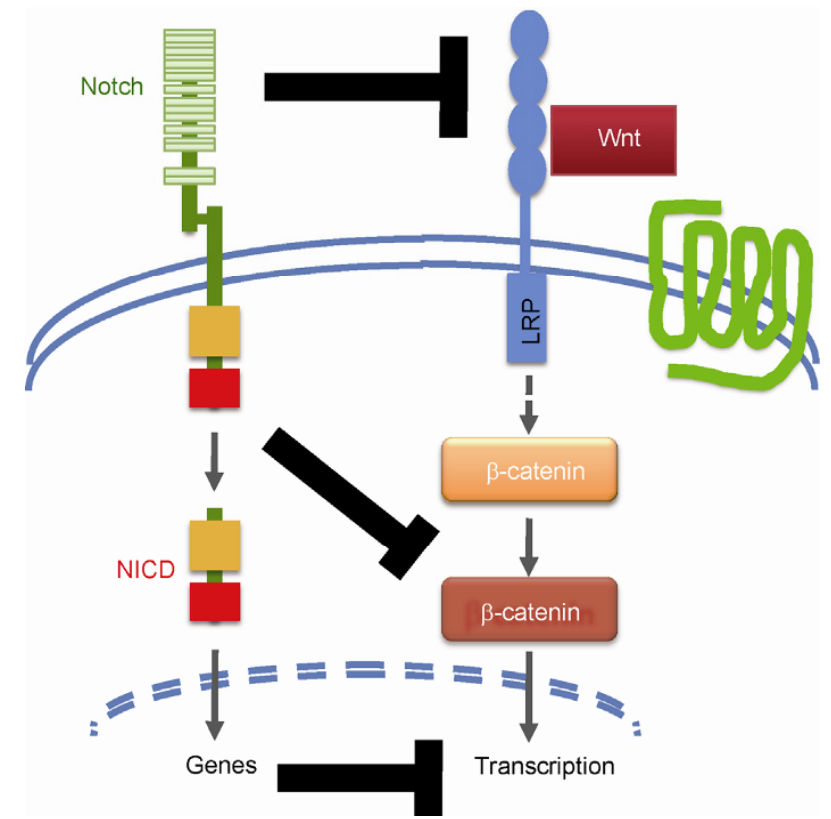

Figure 3 A diagram of the interaction between the Notch and Wnt pathways.

\section{Aberrant Notch and Wnt signaling in cancer}

Given the importance of stem cell signaling pathways in embryonic development, it is not surprising that genetic mutations that perturb their activities result in growth abnormalities in humans. Indeed, many physiological homeostatic activities and pathological responses in adults are related to stem cell functions, thus aberrations in the pathways that regulate stem cells can result in diseases. Among the human diseases most affected by deregulated stem cell signaling are those associated with tissue damage and repair, such as cancer, fibrosis, degenerative diseases and skeletal repair.

\section{The Notch pathway in cancer}

Notch-1 was first identified from a translocation in a subset of T-cell acute leukemia (T-ALL), which fuses the Notch-1 gene to the $\mathrm{T}$-cell receptor- $\beta$ locus, resulting in a fusion protein that is constitutively active [11]. The resultant constitutive Notch-1 signaling is the causative driver of this rare form of T-ALL. More recent work showed that greater than $50 \%$ of all T-ALL patients bear activating point mutations in Notch-1 [12].

On the other hand, Notch-1 can also function as a tumor suppressor. In chronic myelomonocytic leukemia (CMML), inactivating mutations of Notch-1 have been identified, indicating a tumor-suppressive role for Notch signaling [13]. In solid tumors, the role of Notch-1 is not as well characterized, however, evidence for a dual oncogene/tumor suppressor role has emerged [14]. Oncogenic Notch has been reported in breast epithelial tumors and is believed to have a role in anti-estrogen resistance [15]. On the contrary, high frequency of loss-of-function (LoF) mutations in Notch-1 have been discovered by several genome sequencing projects of squamous type carcinomas from the skin, head/neck, and lung [16]. These results suggest that the role of Notch signaling in cancer biology is cell and tissue contextdependent.

\section{The Wnt pathway in cancer}

A possible role for the Wnt pathway in cancer was first discovered in the 1980s in mouse models of breast cancer. Overexpression of Wnt1, induced by a viral insertion at the Wntl locus, was shown to cause spontaneous mammary hyperplasia and tumors in mice [17]. Further studies found that Wnt1, as well as that of other Wnts, promoted the stabilization of free pools of $\beta$-catenin (CTNNB1) and the activation of CTNNB1-dependent transcription [18]. Moreover, inherited inactivating mutations in adenomatous polyposis coli (APC), part of the destruction complex which nega- 
tively regulates the WNT pathway, are found in patients with familial adenomatous polyposis, which can progress to colorectal carcinomas. In line with these data, in 1997, seminal studies demonstrated a crucial role for hyperactivated WNT-CTNNB1 signaling in human colorectal cancers with frequent APC and CTNNB1 mutations [19,20]. Strikingly, activating CTNNB1 mutations or inactivating APC mutations occur in more than $80 \%$ of patients with colorectal cancer. Moreover, an additional $10 \%$ of colorectal tumors harbor fusion mutations of two other Wnt pathway genes encoding R-spondin-2 and R-spondin-3, enhancers of the Wnt pathway [21]. These observations establish that the unregulated activation of the Wnt pathway is the most important oncogenic event in colorectal tumorigenesis.

In addition to colorectal cancer, several other types of cancer also carry mutations in the WNT pathway. For example, liver cancer has an approximately $25 \%$ chance of gaining activating mutations in CTNNB1 or APC, while gastric cancer has an approximately $20 \%$ chance (Sanger Cancer Genome Project database, http://cancer.sanger.ac. uk/cancergenome/projects/cosmic/). Of note, liver cancer and gastric cancer, along with colorectal cancer, are some of the most prevalent types of cancer in China and other parts of the world.

\section{Drug discovery for the notch and Wnt path- ways}

Drug discovery based on the Wnt and Hedgehog pathways has been one of the most active areas in the drug discovery field over the past decade. Many pharmaceutical companies and research institutes have been actively searching for both small molecule and large molecule drugs that target these pathways. Recently, remarkable progress has been made in the search for new drugs.

\section{Targeting the Notch pathway for cancer}

Given the importance of the Notch pathway in many types of cancer, it is not surprising that the discovery of drugs that target this pathway is currently actively pursued by both the pharmaceutical and biotech industries. Several feasible targets have been the focus of these efforts. As shown in Table 1, the first group of compounds is inhibitors of the $\gamma$-secretase complex that process Notch to generate NCID. Several antibodies against either the ligand DLL-4 or the receptors Notch-1, -2 or -3 are also in clinical development for the treatment of T-ALL and certain solid tumors. Notably, all of these compounds are inhibitors of the Notch pathway, which would be useful for certain cancers with activating, but not inactivating, mutations.
Table 1 Notch pathway inhibitors in clinical trials for cancer

\begin{tabular}{lll}
\hline Compound name & Target & Company \\
\hline BMS-906024 & $\gamma$-secretase & BMS \\
MK0752 & $\gamma$-secretase & Merck \\
PF-03084014 & $\gamma$-secretase & Pfizer \\
RO4929097 & $\gamma$-secretase & Roche \\
Enoticumab (REGN421) & DLL-4 & Regeneron \\
MEDI0639 & DLL-4 & MedImmune \\
Demcizumab (OMP-21M18) & DLL-4 & OncoMed \\
OMP-59R5 & Notch-2/3 & OncoMed \\
OMP-52M51 & Notch-1 & OncoMed \\
\hline
\end{tabular}

\section{Targeting the Wnt pathway for cancer}

Given the prevalence of mutations in APC and $\beta$-catenin in many types of cancer, the drug industry has been searching for the elusive inhibitor that blocks $\beta$-catenin transactivation. However, because of a lack of an appropriate target downstream of APC or $\beta$-catenin, this approach has failed. Currently, the industry focus is on the identification of appropriate upstream targets. To this end, several promising programs have entered clinical trials over the past two to three years (Table 2).

One of the most advanced clinical programs is an anti-Frizzled antibody from OncoMed Pharmaceuticals that entered into Phase I clinical trials in 2011. Published data show that this pan-Frizzled antibody (OMP18R5) has remarkable anti-tumor efficacy in many preclinical patient-derived xenograft models [22]. Similar to OMP18R5, OncoMed has several other WNT pathway inhibitors in their pipeline, including a Frizzled-8 Fc fusion protein and an antibody against R-spondin/LGR5 interaction.

Also in Phase I clinical trials is a small molecule inhibitor of Porcupine, an acetyl-transferase responsible for the lipidation, and hence the bioactivity, of all WNT proteins. The molecule, LGK974, is developed by Novartis and currently being tested against multiple types of cancer (http://clinicaltrials.gov/show/NCT01351103).

Several WNT inhibitors have also been pursued in preclinical studies. For example, by screening for agents that specifically kill cancer stem cells, Weinberg, Lander, and colleagues discovered compounds that are selectively toxic to cancer stem cells isolated from breast cancers. One compound, salinomycin, inhibited mammary tumor growth in vivo and induced increased epithelial differentiation of tumor stem cells [23]. A subsequent study by another group demonstrated that salinomycin is a potent inhibitor of the Wnt signaling cascade and acts by interfering with the phosphorylation of LPR6 which is a Wnt receptor [24]. The compound was once pursued by the biotech company Verastem in a preclinical study for the development of a drug targeting breast cancer. 
Table 2 Wnt pathway inhibitors in clinical trials for cancer

\begin{tabular}{lll}
\hline Compound name & Target & Company \\
\hline LGK974 & Porcupine & Novartis \\
Vantictumab (OMP-18R5) & Fzd7/pan Fzds & OncoMed \\
OMP-54F28 & Fzd8 (Fzd8-Fc fusion) & OncoMed \\
OTSA101 & Fzd10 & Centre Leon Berard \\
\hline
\end{tabular}

\section{Targeting the crosstalk between the Notch and Wnt pathways}

As described previously, Notch-1 can be a tumor suppressor in certain types of cancers, such as CMML and squamous type carcinomas from the skin, head-neck, and lung. Large-scale cancer genome projects have revealed loss-of-function mutations of Notch-1 in a large number of squamous carcinomas from the head-neck region and upper aerodigestive tract, including the oral cavity, oropharynx, larynx, thyroid, neck, esophagus, and lung (data available at the Sanger database http://cancer.sanger.ac.uk/cosmic/gene/ overview? In=NOTCH1; ICGC http://dcc.icgc.org/genes/ ENSG00000148400; and TCGA https://tcga-data.nci.nih. gov/tcga/). Notably, cancers that originate from these anatomic sites, such as nasopharyngeal cancer and esophageal cancer, are particularly prevalent in areas of China.

Logically, tumor suppressors are difficult or impossible to target by conventional therapeutic modalities because the loss of a gene cannot be easily replaced in patients. Thus, the LoF mutation of Notch-1 would be impossible to target directly by using small molecule compounds or antibodies. Fortunately, it is still theoretically possible to indirectly target pathways where tumor suppressors are involved. Such approaches include (i) targeting the downstream components of the pathway; (ii) targeting by synthetic lethality, for example, through inhibiting another pathway that interacts with the tumor suppressor. Given the aforementioned notion that the Notch pathway might negatively control the Wnt pathway, i.e., LoF of Notch-1 indirectly activates the Wnt pathway; a plausible approach to target Notch-1 negative tumors would be to inhibit Wnt signaling.

A recent publication by Liu et al. [25] suggested that this just may be correct. In this study, Liu and co-workers from Novartis screened hundreds of cancer cell lines for their sensitivity to the Wnt pathway inhibitor LGK974. They found that a number of head-neck cancer cell lines were sensitive to LGK974. Interestingly, all of the sensitive cell lines harbored Notch-1 mutations that were predicted to be LoF mutations. In xenograft models of HN30 and FaDu (both derived from nasopharyngeal tumors), LGK974 dramatically inhibited the growth of the tumors at doses well-tolerated in mice. This study is consistent with previous observations in terms of the interaction between the two pathways and strongly supports clinical testing of Wnt pathway inhibitors in a large number of cancer patients with
Notch-1 LoF mutations.

\section{Conclusion}

Stem cell research offers great promise not only for cell replacement therapy but also for drug discovery. Over the past few years, remarkable progress has been made in the search for drugs that target two of the most important stem cell pathways, Notch and Wnt. By applying advances in stem cell signaling biology and cancer genomics, drug discovery in this field has become very active. Of particular interest to the Chinese pharmaceutical and research communities is the frequency of Notch mutations in cancer types prevalent in China and the therapeutic potential of WNT inhibitors for their treatment. The continued advancement of this area of research and drug discovery may thus benefit a large number of cancer patients in China.

1 Andersson ER, Sandberg R, Lendahl U. Notch signaling: simplicity in design, versatility in function. Development, 2011, 138: 35933612

2 Koch U, Lehal R, Radtke F. Stem cells living with a notch. Development, 2013, 140: 689-704

3 Nusslein-Volhard C, Wieschaus E. Mutations affecting segment number and polarity in Drosophila. Nature, 1980, 287: 795-801

4 Rijsewijk F, Schuermann M, Wagenaar E, Parren P, Weigel D, Nusse R. The Drosophila homolog of the mouse mammary oncogene int-1 is identical to the segment polarity gene wingless. Cell, 1987, 50: 649-657

5 Clevers H, Nusse R. Wnt/beta-catenin signaling and disease. Cell, 2012, 149: 1192-1205

6 Axelrod JD, Matsuno K, ArtavanisTsakonas S, Perrimon N. Interaction between wingless and Notch signaling pathways mediated by dishevelled. Science, 1996, 271: 1826-1832

7 Estrach S, Ambler CA, Lo Celso C, Hozumi K, Watt FM. Jagged 1 is a beta-catenin target gene required for ectopic hair follicle formation in adult epidermis. Development, 2006, 133: 4427-4438

8 Kwon C, Cheng P, King IN, et al. Notch post-translationally regulates beta-catenin protein in stem and progenitor cells. Nat Cell Biol, 2011, 13: 1244-1251

9 Proweller A, Tu LL, Lepore JJ, Cheng L, Lu MM, Seykora J, Millar SE, Pear WS, Parmacek MS. Impaired notch signaling promotes de novo squamous cell carcinoma formation. Cancer Res, 2006, 66: 7438-7444

10 Nicolas M, Wolfer A, Raj K, Kummer JA, Mill P, van Noort M, Hui CC, Clevers H, Dotto GP, Radtke F. Notch1 functions as a tumor suppressor in mouse skin. Nat Genet, 2003, 33: 416-421

11 Ellisen LW, Bird J, West DC, Soreng AL, Reynolds TC, Smith SD, Sklar J. TAN-1, the human homolog of the Drosophila notch gene, is broken by chromosomal translocations in T lymphoblastic neoplasms. Cell, 1991, 66: 649-661

12 Weng AP, Ferrando AA, Lee W, Morris JP 4th, Silverman LB, Sanchez-Irizarry C, Blacklow SC, Look AT, Aster JC. Activating mutations of NOTCH1 in human T cell acute lymphoblastic leukemia. Science, 2004, 306: 269-271

13 Klinakis A, Lobry C, Abdel-Wahab O, Oh P, Haeno H, Buonamici S, van De Walle I, Cathelin S, Trimarchi T, Araldi E, Liu C, Ibrahim S, Beran M, Zavadil J, Efstratiadis A, Taghon T, Michor F, Levine RL, Aifantis I. A novel tumour-suppressor function for the Notch pathway in myeloid leukaemia. Nature, 2011, 473: 230-233

14 Ranganathan P, Weaver KL, Capobianco AJ. Notch signalling in 
solid tumours: a little bit of everything but not all the time. Nat Rev Cancer, 2011, 11: 338-351

15 Hao L, Rizzo P, Osipo C, Pannuti A, Wyatt D, Cheung LW, Sonenshein G, Osborne BA, Miele L. Notch-1 activates estrogen receptor-alpha-dependent transcription via IKKalpha in breast cancer cells. Oncogene, 2010, 29: 201-213

16 Wang NJ, Sanborn Z, Arnett KL, Bayston LJ, Liao W, Proby CM, Leigh IM, Collisson EA, Gordon PB, Jakkula L, Pennypacker S, Zou Y, Sharma M, North JP, Vemula SS, Mauro TM, Neuhaus IM, Leboit PE, Hur JS, Park K, Huh N, Kwok PY, Arron ST, Massion PP, Bale AE, Haussler D, Cleaver JE, Gray JW, Spellman PT, South AP, Aster JC, Blacklow SC, Cho RJ. Loss-of-function mutations in Notch receptors in cutaneous and lung squamous cell carcinoma. Proc Natl Acad Sci USA, 2011, 108: 17761-17766

17 Nusse R, Varmus HE. Many tumors induced by the mouse mammary-tumor virus contain a provirus integrated in the same region of the host genome. Cell, 1982, 31: 99-109

18 Papkoff J, Rubinfeld B, Schryver B, Polakis P. Wnt-1 regulates free pools of catenins and stabilizes APC-catenin complexes. Mol Cell Biol, 1996, 16: 2128-2134

19 Morin PJ, Sparks AB, Korinek V, Barker N, Clevers H, Vogelstein B, Kinzler KW. Activation of beta-catenin-Tcf signaling in colon cancer by mutations in beta-catenin or APC. Science, 1997, 275: 1787-1790

20 Korinek V, Barker N, Morin PJ, van Wichen D, de Weger R, Kinzler $\mathrm{KW}$, Vogelstein B, Clevers H. Constitutive transcriptional activation by a beta-catenin-Tcf complex in $\mathrm{APC}^{-/-}$colon carcinoma. Science, 1997, 275: 1784-1787

21 Seshagiri S, Stawiski EW, Durinck S, Modrusan Z, Storm EE, Conboy CB, Chaudhuri S, Guan Y, Janakiraman V, Jaiswal BS, Guillory J, Ha C, Dijkgraaf GJ, Stinson J, Gnad F, Huntley MA, Degenhardt JD, Haverty PM, Bourgon R, Wang W, Koeppen H, Gentleman R, Starr TK, Zhang Z, Largaespada DA, Wu TD, de Sauvage FJ. Recurrent R-spondin fusions in colon cancer. Nature, 2012, 488: 660-664

22 Gurney A, Axelrod F, Bond CJ, Cain J, Chartier C, Donigan L, Fischer M, Chaudhari A, Ji M, Kapoun AM, Lam A, Lazetic S, Ma S, Mitra S, Park IK, Pickell K, Sato A, Satyal S, Stroud M, Tran H, Yen WC, Lewicki J, Hoey T. Wnt pathway inhibition via the targeting of Frizzled receptors results in decreased growth and tumorigenicity of human tumors. Proc Natl Acad Sci USA, 2012, 109: 11717-11722

23 Gupta PB, Onder TT, Jiang GZ, Tao K, Kuperwasser C, Weinberg RA, Lander ES. Identification of selective inhibitors of cancer stem cells by high-throughput screening. Cell, 2009, 138: 645-659

24 Lu DS, Choi MY, Yu J, Castro JE, Kipps TJ, Carson DA. Salinomycin inhibits Wnt signaling and selectively induces apoptosis in chronic lymphocytic leukemia cells. Proc Natl Acad Sci USA, 2011, 108: $13253-13257$

25 Liu J, Pan SF, Hsieh MH, et al. Targeting Wnt-driven cancer through the inhibition of Porcupine by LGK974. Proc Natl Acad Sci USA, 2013, 110: 20224-20229

Open Access This article is distributed under the terms of the Creative Commons Attribution License which permits any use, distribution, and reproduction in any medium, provided the original author(s) and source are credited. 